\title{
ACS Combinatorial Science
}

\author{
Supporting Information
}

\section{Polymer Microarrays for the Discovery and Optimization of Robust Optical Fibre-based pH Sensors}

Jingjing Gong ${ }^{\dagger, £}$, Seshasailam Venkateswaran ${ }^{\dagger}$, Michael G Tanner ${ }^{\dagger, £}$, James M Stone $^{\S, £}$, Mark Bradley*,†,£

†School of Chemsitry, EaStCHEM, University of Edinburgh, King’s Buildings, West Mains Road, Edinburgh, EH9 3FJ, UK

\#nstitute of Photonics and Quantum Sciences, School of Engineering and Physical Sciences, HeriotWatt University, Edinburgh, EH14 4AS, UK

${ }^{\S}$ Centre for Photonics and Photonic Materials, Department of Physics, University of Bath, Bath, BA2 7AY, UK

${ }^{\mathfrak{E}}$ EPSRC Proteus Hub, Centre for Inflammation Research, Queen's Medical Research Institute, University of Edinburgh, 47 Little France Crescent, Edinburgh, EH16 4TJ, UK 


\section{Abbreviations}

MMA

DMAEA

DEAA

PAA

MEMA

DMAA

HEMA

HPMA

HBMA

DEAEMA

DEAEA

MTEMA

BAEMA

DMAPMAA

VAA

VI

VPNO

VP-4

VP-2

DAAA

BACOEA

A-H

MA-H
Methyl methacrylate

dimethylaminoethyl acrylate

$\mathrm{N}, \mathrm{N}$-diethylacetoacetamide

polyacrylic acid

2-methoxyethyl methacrylate

dimethylacrylamide

2-hydroxyethyl methacrylate

N-(2-Hydroxypropyl) methacrylamide

4-hydroxybutyl methacrylate

2-(diethylamino)ethyl methacrylate

2-(diethylamino)ethyl acrylate

(methylthio) ethyl methacrylate

t-(butylamino)ethyl methacrylate

$\mathrm{N}$-[3-(dimethylaminopropyl)] methacrylamide

vinylacetic acid

1-vinylimidazol

1-vinyl-2-pyrrolidinone

4-Vinyl pyridine

2-Vinyl pyridine

diacetone acrylamide

2-[[(butylamino)carbonyl]oxy]ethyl acrylate

acrylic acid

methacrylic acid 
EGMP

EMA

BMA

DMAEMA

GMA ethylene glycol methacrylate phosphate

ethyl methacrylate

N-butyl methacrylate

2-(dimethylamino)ethyl methacrylate

glycidyl methacrylate 


\section{PA101 synthesis and characterization}

The hit polymer PA101 was resynthesized by free-radical polymerisation, using 2,2'azobis(2-methylpropionitrile) (AIBN) as an initiator. Reaction conditions are given in Table S1. The monomers, AIBN and the solvent were mixed in a glass vessel, and polymerisation carried out for $48 \mathrm{~h}$ under a $\mathrm{N}_{2}$ atm. The polymers were precipitated by the dropwise addition of the reaction mixture into hexane, collected by filtration, washed hexane, and dried overnight in vacuo at $40^{\circ} \mathrm{C}$. The polymer PA101 was characterised by GPC (Table S2), NMR (Figure S1) and FT-IR (Figure S2).

Table S1. Synthesis of PA101.

\begin{tabular}{|c|c|c|c|c|c|c|}
\hline Polymer & \multicolumn{2}{|c|}{ Monomers } & AIBN & Solvent & T & Yield \\
\hline PA101 & MMA & DMAEA & $0.0625 \mathrm{mmol}$ & Toluene & $60^{\circ} \mathrm{C}$ & $89 \%$ \\
& & & & $(6 \mathrm{~mL})$ & & \\
& $45 \mathrm{mmoL}$ & $5 \mathrm{mmoL}$ & & & & \\
\hline
\end{tabular}

Table S2. Molecular weight (Mw and Mn) and polydispersity index (PDI) of PA101.

\begin{tabular}{cccc}
\hline Polymer & Mw & Mn & PDI \\
& & & \\
\hline PA101 & $226 \mathrm{KDa}$ & $140 \mathrm{KDa}$ & 1.6 \\
& & & \\
\hline
\end{tabular}




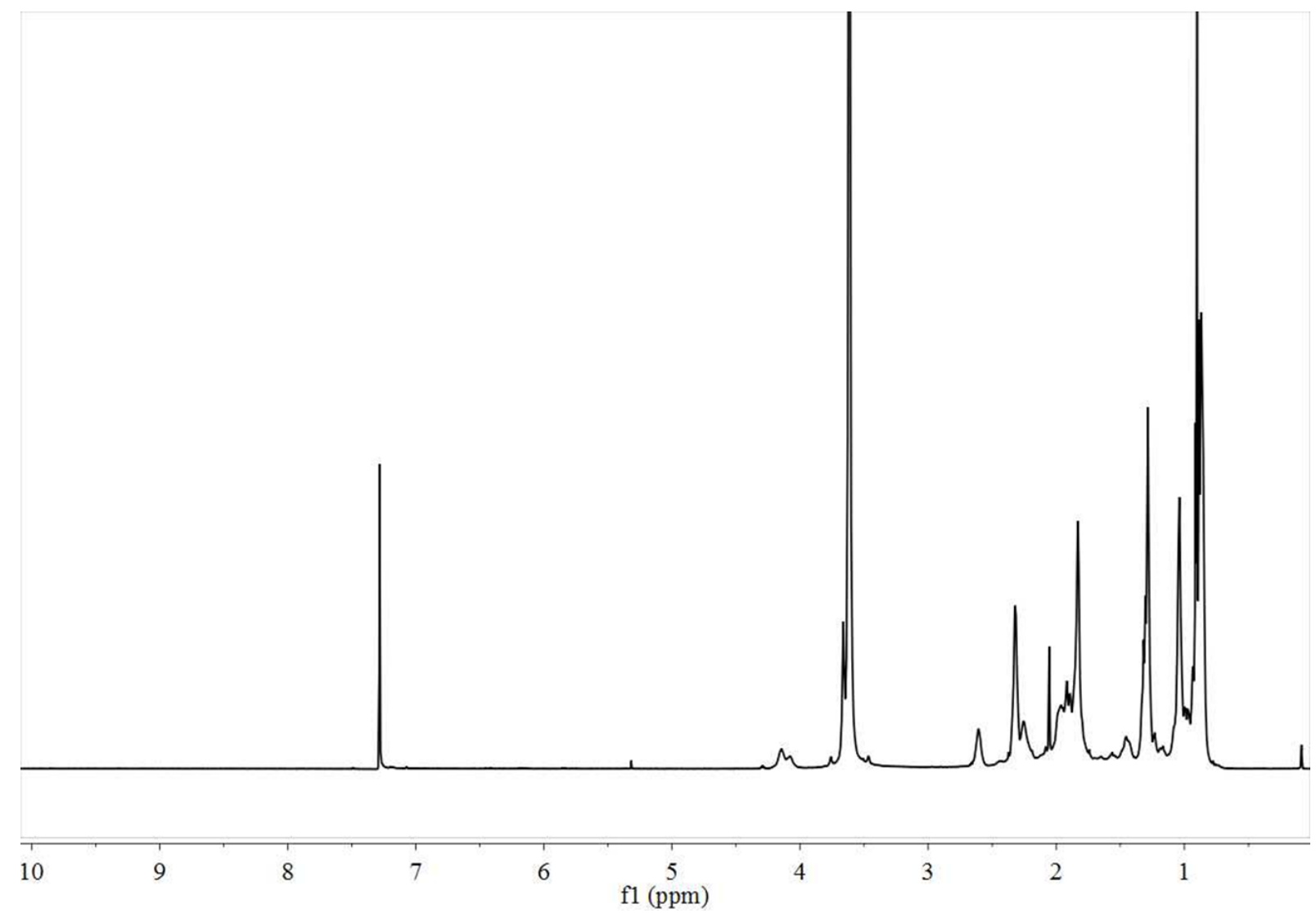

Figure S1. NMR of PA101 


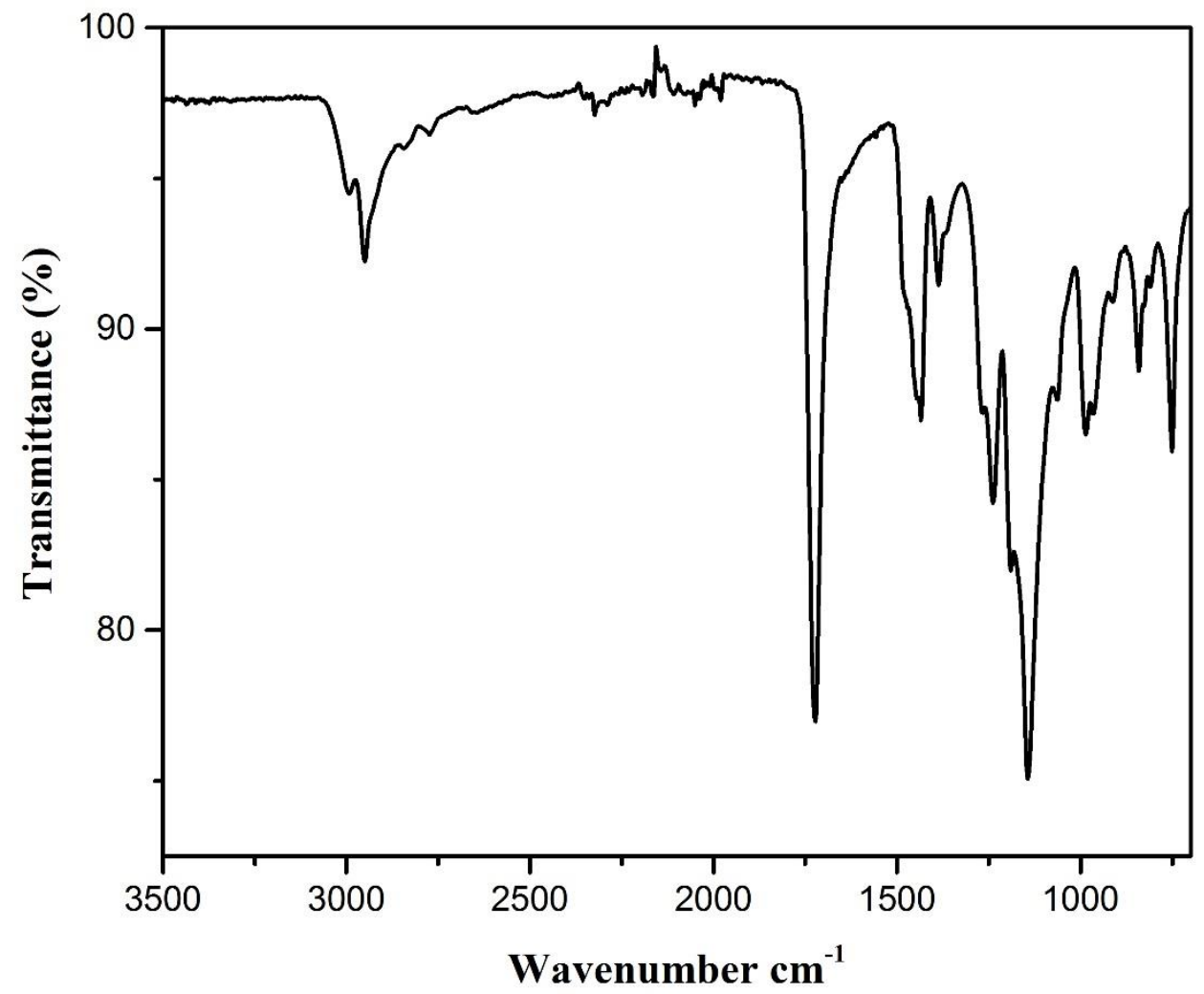

Figure S2. FT-IR of PA101 
Table. S3. Fold increase in fluorescence for the polymer spots mixed with 5(6)-carboxyfluorescein when changing $\mathrm{pH}$ buffer from $\mathrm{pH} 4.0$ to 10.0 . The fold increase was calculated from the average of 4 microarray slides. $A=0.025 \% 5(6)$-carboxyfluorescein, $B=0.01 \% 5(6)$-carboxyfluorescein, $\mathrm{C}=0.04 \%$ 5(6)-carboxyfluorescein.

\begin{tabular}{|c|c|c|c|c|c|c|c|}
\hline Polymer & rMonomer 1 & Monomer 2 & Monomer 3 & $\begin{array}{l}\text { Ratio o } \\
\text { 3Monomers }\end{array}$ & $\begin{array}{l}\text { ff Fold } \\
\text { increase (A) }\end{array}$ & $\begin{array}{l}\text { Fold } \\
\text { increase (B) }\end{array}$ & $\begin{array}{l}\text { Fold } \\
\text { increase }(C)\end{array}$ \\
\hline PA1 & St & DEAA & & $90: 10$ & 1.24 & 1.82 & 2.50 \\
\hline PA2 & St & DEAA & & $70: 30$ & 1.15 & 1.74 & 2.22 \\
\hline PA3 & St & DEAA & & $50: 50$ & 1.16 & 1.60 & 1.80 \\
\hline PA4 & St & PAA & & $90: 10$ & 1.18 & 1.65 & 2.66 \\
\hline PA5 & St & PAA & & $70: 30$ & 1.30 & 1.09 & 2.77 \\
\hline PA6 & MMA & DEAA & & $90: 10$ & 1.25 & 1.47 & 1.85 \\
\hline PA7 & MMA & DEAA & & $70: 30$ & 1.23 & 1.60 & 2.30 \\
\hline PA8 & MMA & DMAA & & $90: 10$ & 1.30 & 1.71 & 3.02 \\
\hline PA9 & MMA & DMAA & & $70: 30$ & 1.20 & 1.73 & 3.06 \\
\hline PA10 & MMA & DEAA & & $50: 50$ & 1.21 & 1.79 & 2.67 \\
\hline PA11 & MMA & PAA & & $90: 10$ & 1.24 & 1.67 & 2.55 \\
\hline PA12 & MMA & PAA & & $70: 30$ & 1.36 & 1.81 & 2.83 \\
\hline PA13 & MMA & PAA & & $50: 50$ & 1.16 & 1.92 & 3.54 \\
\hline PA14 & MEMA & DEAA & & $90: 10$ & 1.25 & 1.52 & 2.12 \\
\hline PA15 & MEMA & DEAA & & $70: 30$ & 1.20 & 1.65 & 2.16 \\
\hline PA16 & MEMA & DEAA & & $50: 50$ & 1.18 & 1.62 & 2.95 \\
\hline PA17 & MEMA & DMAA & & $90: 10$ & 1.33 & 1.85 & 2.82 \\
\hline PA18 & MEMA & DMAA & & $70: 30$ & 1.21 & 1.37 & 1.78 \\
\hline PA19 & MEMA & DMAA & & $50: 50$ & 1.18 & 1.36 & 1.65 \\
\hline PA20 & MEMA & PAA & & $90: 10$ & 1.18 & 1.42 & 1.73 \\
\hline PA21 & MEMA & PAA & & $70: 30$ & 1.23 & 1.56 & 1.85 \\
\hline PA22 & MEMA & PAA & & $50: 50$ & 1.17 & 1.64 & 2.63 \\
\hline PA23 & MEA & PAA & & $50: 50$ & 1.10 & 1.53 & 2.49 \\
\hline
\end{tabular}




\begin{tabular}{|c|c|c|c|c|c|c|}
\hline PA24 & HEMA & DEAA & $70: 30$ & 1.13 & 1.51 & 2.08 \\
\hline PA25 & HEMA & DMAA & $90: 10$ & 1.17 & 1.40 & 2.00 \\
\hline PA26 & HEMA & DMAA & $70: 30$ & 1.21 & 1.62 & 2.50 \\
\hline PA27 & HРMA & DEAA & $50: 50$ & 1.08 & 1.51 & 2.82 \\
\hline PA28 & НРMA & DMAA & $50: 50$ & 1.18 & 1.40 & 1.84 \\
\hline PA29 & HBMA & DMAA & $70: 30$ & 1.23 & 1.50 & 1.87 \\
\hline PA30 & HBMA & DMAA & $50: 50$ & 1.21 & 1.49 & 1.56 \\
\hline PA31 & HBMA & PAA & $90: 10$ & 1.31 & 1.46 & 3.24 \\
\hline PA32 & HBMA & PAA & $70: 30$ & 1.23 & 1.84 & 2.62 \\
\hline PA33 & HBMA & PAA & $50: 50$ & 1.38 & 1.83 & 2.69 \\
\hline PA34 & MEMA & DEAEMA & $50: 50$ & 1.33 & 1.46 & 2.60 \\
\hline PA35 & MEMA & DMAEMA & 90:10 & 1.50 & 1.81 & 2.31 \\
\hline PA36 & MEMA & DMAEMA & $70: 30$ & 2.36 & 2.82 & 4.23 \\
\hline PA37 & MEMA & DMAEMA & $50: 50$ & 2.33 & 2.23 & 3.95 \\
\hline PA38 & MEMA & DEAEA & $70: 30$ & 1.43 & 1.88 & 3.58 \\
\hline PA39 & MEMA & MTEMA & $70: 30$ & 1.36 & 1.60 & 2.53 \\
\hline PA40 & MEMA & MTEMA & $50: 50$ & 1.34 & 1.65 & 2.01 \\
\hline PA41 & MEMA & BAEMA & $90: 10$ & 1.60 & 2.05 & 2.93 \\
\hline PA42 & MEMA & BAEMA & $70: 30$ & 1.35 & 1.89 & 2.89 \\
\hline PA43 & MEMA & BAEMA & $50: 50$ & 1.58 & 2.45 & 4.77 \\
\hline PA44 & MEMA & DMAPMAA & 90:10 & 1.41 & 2.43 & 3.50 \\
\hline PA45 & MEMA & VAA & $90: 10$ & 1.24 & 1.44 & 2.42 \\
\hline PA46 & MEMA & VAA & $70: 30$ & 1.18 & 1.52 & 2.07 \\
\hline PA47 & MEMA & VAA & $50: 50$ & 1.24 & 1.79 & 2.17 \\
\hline PA48 & MEMA & VI & $70: 30$ & 1.20 & 1.46 & 2.29 \\
\hline PA49 & MEMA & VPNO & $90: 10$ & 1.26 & 1.53 & 2.81 \\
\hline PA50 & MEMA & VPNO & $70: 30$ & 1.24 & 1.50 & 2.86 \\
\hline PA51 & MEMA & VPNO & $50: 50$ & 1.15 & 1.27 & 2.34 \\
\hline PA52 & MEMA & VP-4 & $90: 10$ & 1.19 & 1.75 & 2.45 \\
\hline PA53 & MEMA & VP-4 & $70: 30$ & 1.18 & 1.90 & 2.23 \\
\hline
\end{tabular}




\begin{tabular}{|c|c|c|c|c|c|c|c|}
\hline PA54 & MEMA & VP-4 & & $50: 50$ & 1.13 & 1.60 & 2.55 \\
\hline PA55 & MEMA & VP-2 & & $90: 10$ & 1.27 & 1.76 & 2.32 \\
\hline PA56 & MEMA & VP-2 & & $70: 30$ & 1.15 & 1.70 & 2.67 \\
\hline PA57 & MEMA & VP-2 & & $50: 50$ & 1.45 & 2.19 & 2.97 \\
\hline PA58 & MEMA & DAAA & & $90: 10$ & 1.25 & 1.74 & 2.64 \\
\hline PA59 & MEMA & DAAA & & $70: 30$ & 1.30 & 1.64 & 2.28 \\
\hline PA60 & MEMA & DAAA & & $50: 50$ & 1.32 & 1.76 & 2.68 \\
\hline PA61 & HEMA & DEAEMA & & $50: 50$ & 2.08 & 1.88 & 2.36 \\
\hline PA62 & HEMA & DMAEMA & & $90: 10$ & 1.39 & 2.06 & 3.44 \\
\hline PA63 & HEMA & DMAEMA & & $50: 50$ & 2.54 & 2.49 & 5.95 \\
\hline PA64 & HEMA & MTEMA & & $90: 10$ & 1.26 & 1.62 & 2.28 \\
\hline PA65 & HEMA & BAEMA & & 90:10 & 1.23 & 1.79 & 3.05 \\
\hline PA66 & HEMA & BAEMA & & $70: 30$ & 1.37 & 1.56 & 2.24 \\
\hline PA67 & HEMA & DMAPMAA & & $90: 10$ & 1.32 & 1.88 & 2.86 \\
\hline PA68 & HEMA & DMAPMAA & & $70: 30$ & 1.41 & 2.24 & 4.30 \\
\hline PA69 & HEMA & DMAPMAA & & $50: 50$ & 1.73 & 2.50 & 3.51 \\
\hline PA70 & HEMA & BACOEA & & 90:10 & 1.26 & 1.54 & 2.58 \\
\hline PA71 & HEMA & VAA & & $90: 10$ & 1.26 & 1.78 & 2.66 \\
\hline PA72 & HEMA & VAA & & $70: 30$ & 1.18 & 1.59 & 2.04 \\
\hline PA73 & MMA & A-H & & $70: 30$ & 1.14 & 1.30 & 1.55 \\
\hline PA74 & MMA & MA-H & & $90: 10$ & 1.15 & 1.47 & 2.28 \\
\hline PA75 & MMA & MA-H & & $50: 50$ & 1.20 & 1.47 & 1.74 \\
\hline PA76 & MEMA & MA-H & & 90:10 & 1.10 & 1.44 & 1.72 \\
\hline PA77 & MEMA & MA-H & & $70: 30$ & 1.18 & 1.40 & 2.03 \\
\hline PA78 & MEMA & MA-H & & $50: 50$ & 1.20 & 1.48 & 2.15 \\
\hline PA79 & MEMA & EGMP & & $90: 10$ & 1.16 & 1.26 & 1.45 \\
\hline PA80 & MEMA & EGMP & & $70: 30$ & 1.14 & 1.21 & 1.51 \\
\hline PA81 & MMA & A-H & DEAEMA & $70: 20: 10$ & 1.16 & 1.36 & 1.66 \\
\hline PA82 & MMA & A-H & DEAEA & $70: 20: 10$ & 1.16 & 1.29 & 1.76 \\
\hline PA83 & MMA & A-H & DEAEA & $70: 15: 15$ & 1.13 & 1.31 & 1.48 \\
\hline
\end{tabular}




\begin{tabular}{|c|c|c|c|c|c|c|c|}
\hline PA84 & MMA & MA-H & DEAEA & $70: 20: 10$ & 1.37 & 2.35 & 3.58 \\
\hline PA85 & MMA & MA-H & DEAEA & $70: 15: 15$ & 1.12 & 1.34 & 2.29 \\
\hline PA86 & MMA & MA-H & DEAEA & $70: 10: 20$ & 1.28 & 1.60 & 2.10 \\
\hline PA87 & MEMA & A-H & DEAEMA & $70: 20: 10$ & 1.13 & 1.81 & 2.20 \\
\hline PA88 & MEMA & A-H & DEAEA & 70:20:10 & 1.33 & 1.93 & 3.08 \\
\hline PA89 & MEMA & A-H & DEAEA & $70: 15: 15$ & 1.25 & 1.72 & 2.72 \\
\hline PA90 & MEMA & $\mathrm{A}-\mathrm{H}$ & DEAEA & $70: 10: 20$ & 1.19 & 1.77 & 2.36 \\
\hline PA91 & MEMA & MA-H & DEAEMA & 70:20:10 & 1.18 & 1.80 & 2.53 \\
\hline PA92 & MEMA & MA-H & DEAEMA & $70: 15: 15$ & 1.09 & 1.27 & 1.89 \\
\hline PA93 & MEMA & MA-H & DEAEA & $70: 20: 10$ & 1.35 & 2.02 & 2.94 \\
\hline PA94 & MEMA & MA-H & DEAEA & $70: 15: 15$ & 1.26 & 1.78 & 2.67 \\
\hline PA95 & MEMA & MA-H & DEAEA & $70: 10: 20$ & 1.23 & 1.71 & 2.62 \\
\hline PA96 & MMA & DEAEMA & & $90: 10$ & 1.20 & 2.30 & 2.94 \\
\hline PA97 & MMA & DEAEMA & & $70: 30$ & 1.48 & 2.08 & 3.07 \\
\hline PA98 & MMA & DEAEMA & & $50: 50$ & 2.45 & 3.12 & 3.80 \\
\hline PA99 & MMA & DEAEA & & $90: 10$ & 1.86 & 5.32 & 11.76 \\
\hline PA100 & MMA & DEAEA & & $70: 30$ & 1.22 & 1.62 & 2.01 \\
\hline PA101 & MMA & DMAEA & & $90: 10$ & 2.23 & 5.63 & 14.24 \\
\hline PA102 & MMA & DMAEA & & $70: 30$ & 2.03 & 2.67 & 4.14 \\
\hline PA103 & HPMA & DEAEA & & $70: 30$ & 1.29 & 1.69 & 2.21 \\
\hline PA104 & HBMA & DEAEMA & & $70: 30$ & 1.23 & 1.52 & 2.35 \\
\hline PA105 & EMA & DEAEMA & & $90: 10$ & 1.70 & 1.53 & 2.77 \\
\hline PA106 & EMA & DEAEMA & & $50: 50$ & 1.34 & 1.63 & 2.77 \\
\hline PA107 & EMA & DMAEMA & & $70: 30$ & 1.78 & 2.48 & 2.09 \\
\hline PA108 & EMA & DEAEA & & $90: 10$ & 1.21 & 2.39 & 1.91 \\
\hline PA109 & EMA & DMAEA & & $90: 10$ & 1.14 & 1.74 & 2.67 \\
\hline PA110 & BMA & DEAEMA & & $90: 10$ & 1.33 & 2.04 & 2.25 \\
\hline PA111 & BMA & DMAEMA & & $90: 10$ & 1.39 & 2.07 & 2.86 \\
\hline PA112 & BMA & DMAEA & & $90: 10$ & 1.49 & 2.20 & 2.84 \\
\hline PA113 & MMA & GMA & & $90: 10$ & 1.30 & 1.82 & 2.78 \\
\hline
\end{tabular}




\begin{tabular}{|c|c|c|c|c|c|c|}
\hline PA114 & MMA & GMA & $50: 50$ & 1.96 & 3.20 & 4.06 \\
\hline PA1 15 & MEMA & & $100 \%$ & 1.24 & 2.58 & 2.15 \\
\hline PA1 16 & MMA & & $100 \%$ & 1.22 & 1.53 & 2.13 \\
\hline PA117 & HBMA & & $100 \%$ & 1.20 & 2.16 & 3.86 \\
\hline PA118 & HPMA & & $100 \%$ & 1.28 & 1.46 & 2.03 \\
\hline PA1 19 & BMA & & $100 \%$ & 1.52 & 1.59 & 3.97 \\
\hline PA120 & HEMA & & $100 \%$ & 1.26 & 1.95 & 3.23 \\
\hline PA121 & EMA & & $100 \%$ & 1.26 & 1.81 & 3.62 \\
\hline
\end{tabular}




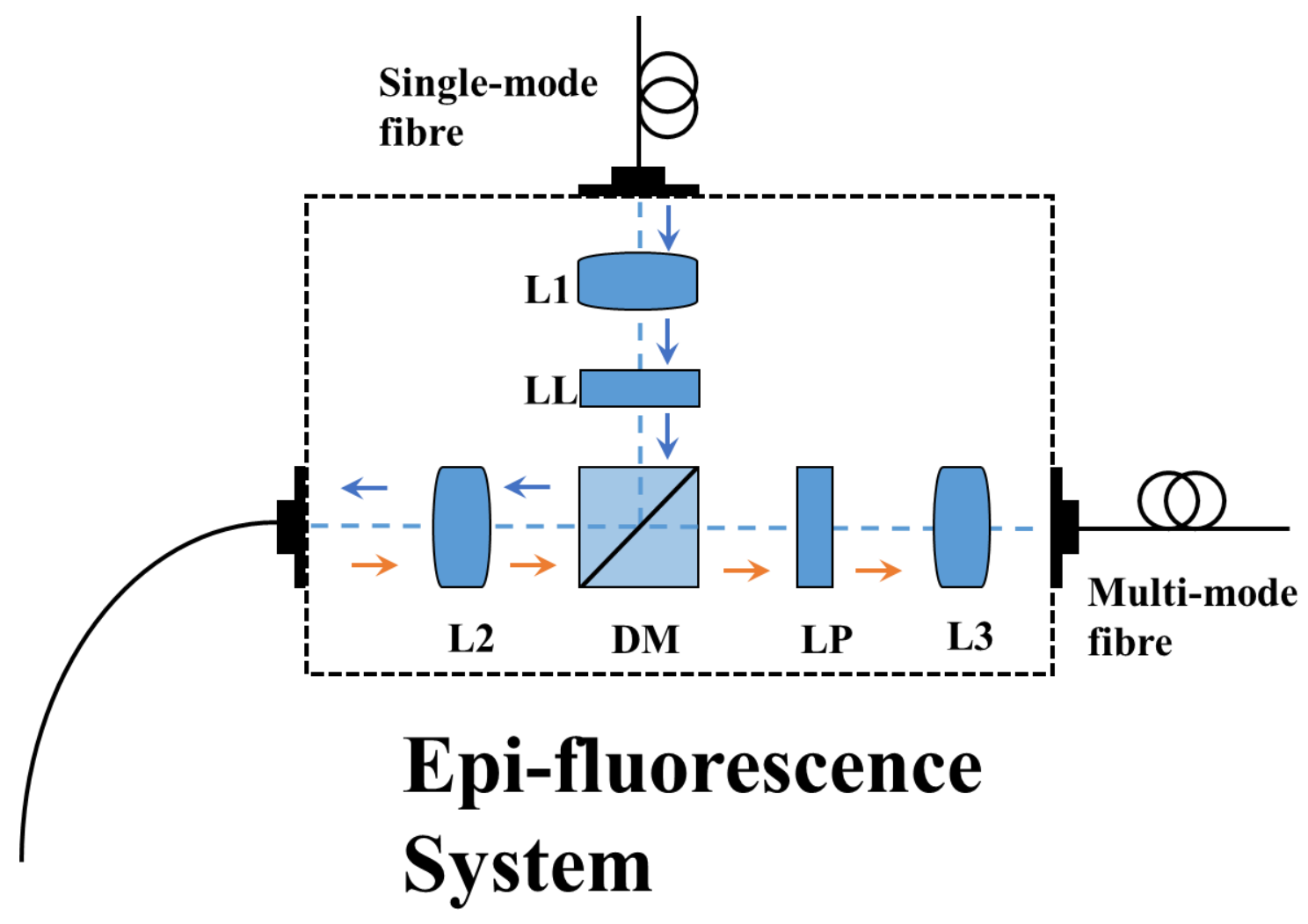

Figure S3. An epi-fluorescence system was used to couple the excitation light into the optical fibre probe with coupling of the excitation fluorescence signal light into the spectrometer. The illuminating light from

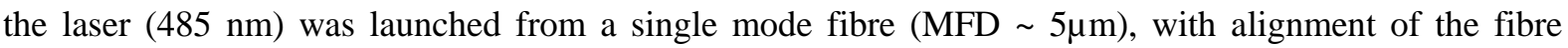
controlled relative to a compact aspheric lens (L1) to provide a collimated on axis beam into the fibre coupling system. After passing through a laser line filter (LL) to ensure a clean spectrum of the desired wavelength, the excitation light was then reflected by a dichroic mirror and focused onto the core of the optical fibre probe using lens L2. The position of the core was noted through imaging of the distal end of the fibre, with the $\mathrm{XY}$ and $\mathrm{Z}$ control of the optical fibre using a translational stage. The fluorescent light emitted from the probe was roughly collimated by L2, and passed straight through the dichroic mirror, with a long-pass filter (LP, cut-on of wavelengths $>510 \mathrm{~nm}$ ). The fluorescent light was coupled into a spectrometer collection multi-mode fibre (50 $\mu \mathrm{m}$ collection fibre) using lens L3. In all cases, compact aspheric lenses (350-700 $\mathrm{nm}$ broadband with an antireflective (AR) coating) were used, with identical lenses were used in all locations to give unity magnification of the laser source. 
(a)

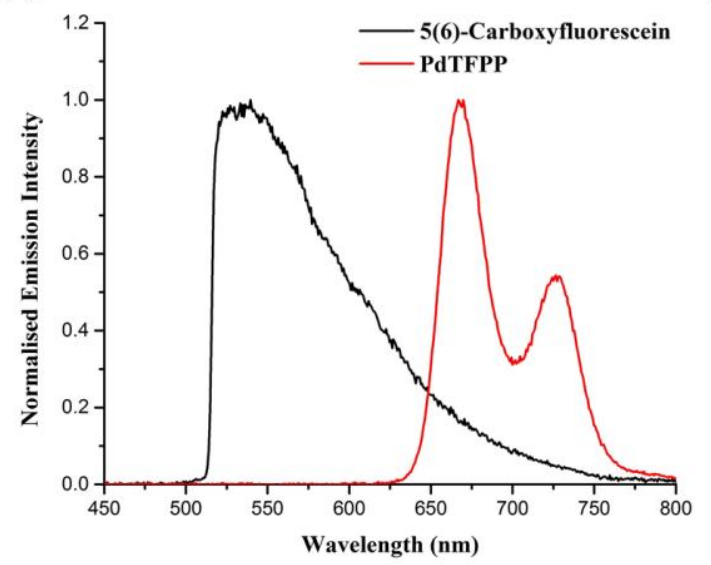

(b)

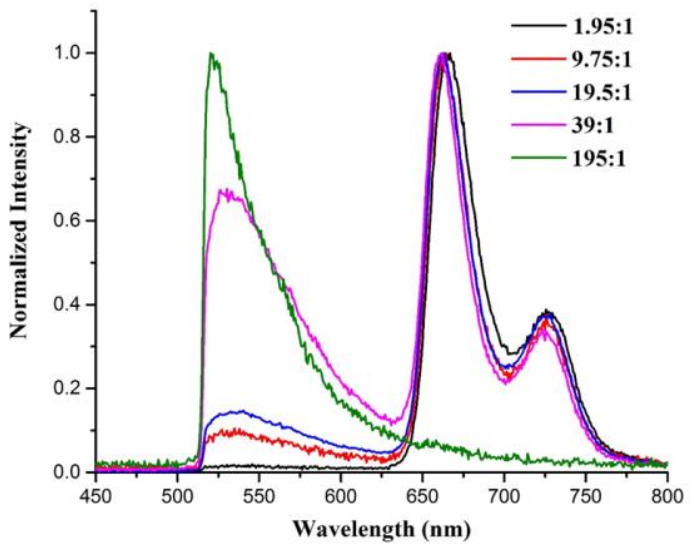

Figure S4. (a) Emission spectra of 5(6)-carboxyfluorescein and PdTFPP and (b) emission spectra of the optical fibre 5(6)-carboxyfluorescein/PdTFPP sensors fabricated with different ratios of 5(6)carboxyfluorescein/PdTFPP. Excitation at $485 \mathrm{~nm}, 1 \mu \mathrm{W}$. The spectra were recorded with an integration time of $100 \mathrm{~ms}$. The sharp leading-edge is due to the excitation filter that cuts off wavelengths $<510 \mathrm{~nm}$. 


\section{Experimental procedures for $\mathrm{pH}$ measurement on the ovine tissue sample}

The fabricated optical fibre probe was calibrated using a range of phosphate buffers ( $\mathrm{pH} 6.0$ to 8.0) before tissue measurements. The fibre tip of the probe was placed in contact with the surface of the tissue and left in place for approximately $1 \mathrm{~min}$, ensuring that the fluorescence spectrum was stable before measurements were taken. Four sample locations were used and at each location three measurements were recorded, with the probe washed between each measurement.

(a)

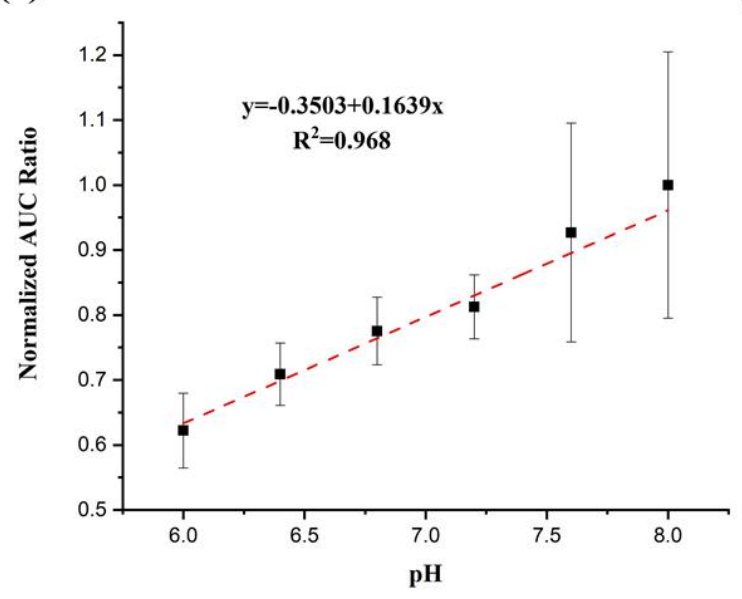

(b)

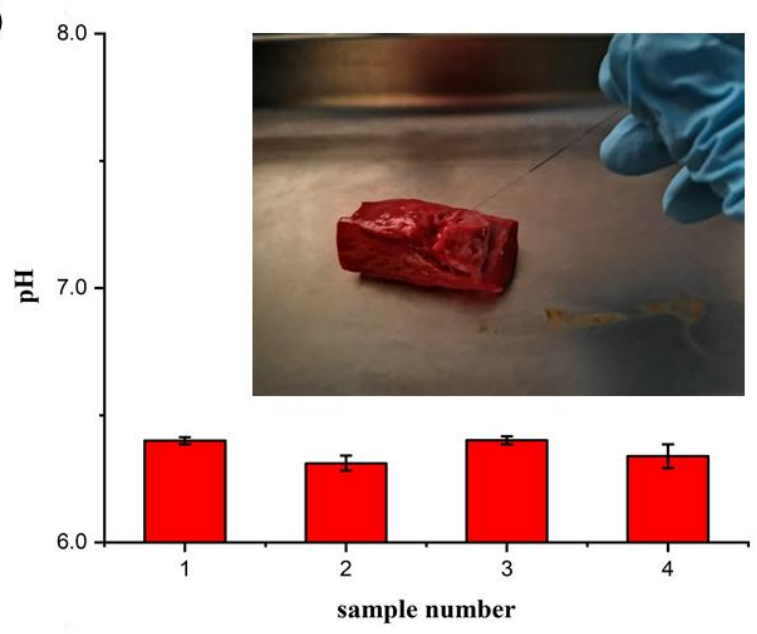

Figure S5. (a) Calibration of the fibre $\mathrm{pH}$ probe prepared for tissue measurement and (b) The tissue $\mathrm{pH}$ variation measured using the fabricated probe at each sample locations. Inset: Example photograph of tissue $\mathrm{pH}$ measurement using the fabricated probe. 


\section{Matlab script for fluorescent sensor distribution analysis on the end of the}

\section{fibre.}

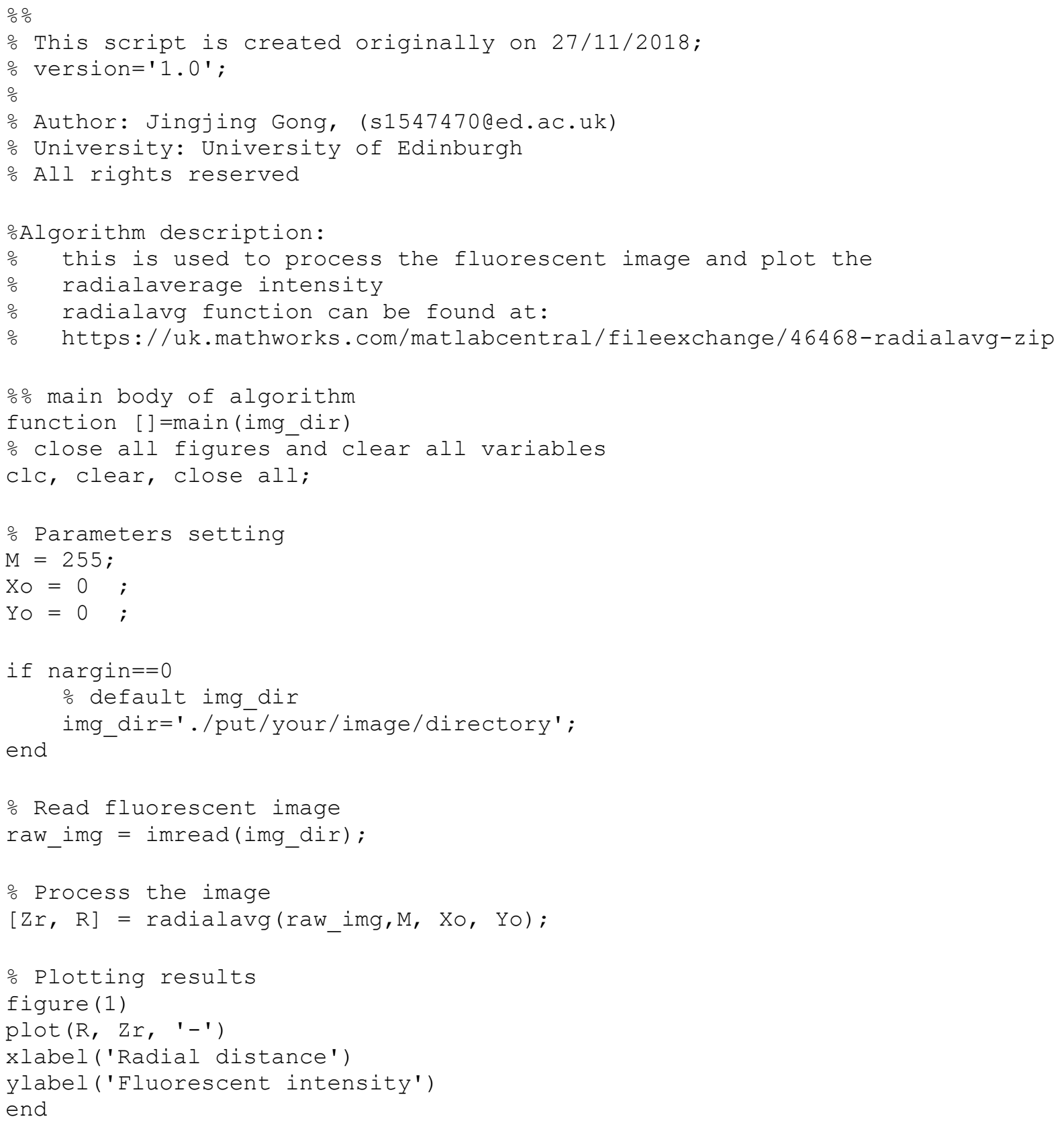

